\title{
Reconstruction of long-period fiber gratings from their core-to-core transmission function
}

\author{
Amir Rosenthal and Moshe Horowitz \\ Department of Electrical Engineering, Technion-Israel Institute of Technology, Haifa 32000, Israel
}

Received March 4, 2005; revised manuscript received June 6, 2005; accepted June 13, 2005

\begin{abstract}
In order to reconstruct the structure of a long-period grating, both the complex core-to-core transmission function and the complex core-to-cladding transmission function should be known. However, in practice, only the core-to-core transmission function of the grating can be measured. We demonstrate theoretically the reconstruction of long-period gratings from only the core-to-core transmission function. The reconstruction is performed by extracting the complex core-to-cladding transmission function of the grating from its core-to-core transmission function. Generally, the extraction is not unique; however, we show that by writing an additional grating in cascade to the interrogated grating, a unique reconstruction can be obtained. In weak long-period gratings, only the amplitude of the core-to-core transmission function is needed to reconstruct the grating. The results of our work can enable the experimental reconstruction of long-period gratings from their transmission function as well as the development of novel distributed sensors. (C) 2006 Optical Society of America

OCIS codes: $050.2770,290.3200,100.5070$.
\end{abstract}

\section{INTRODUCTION}

In recent years, there has been extensive research on the use of long-period fiber gratings (LPGs) and fiber Bragg gratings (FBGs) as spectral filters and as fiber sensors. ${ }^{1-4}$ The complex reflection spectrum of a FBG can be used for uniquely reconstructing the grating profile by using inverse-scattering algorithms. ${ }^{5-8}$ The reconstruction techniques of FBGs have been used for improving the writing process of the gratings and for developing novel distributed fiber Bragg sensors. ${ }^{9-11}$

Inverse-scattering algorithms were also developed for reconstructing the structure of LPGs. ${ }^{12-14}$ These algorithms were used for synthesizing LPGs according to their desired spectral properties. The inverse-scattering algorithms require knowledge of both the complex core-tocore and the complex core-to-cladding transmission spectra. The complex core-to-core transmission spectrum can be simply measured by using techniques such as lowcoherence spectral interferometry. ${ }^{15-17}$ Generally, the core-to-core transmission spectrum does not uniquely define the grating structure, and many different gratings may have the same core-to-core transmission spectrum. ${ }^{14}$ Therefore, the measurement of only the complex core-tocore transmission spectrum is not sufficient for uniquely characterizing LPGs. In principle, the complex core-tocladding transmission spectrum may be measured by interfering the transmitted cladding mode with a reference beam. However, such a measurement would require complicated bulk optics and cannot be performed when the long-period grating is used as a sensor. In Ref. 18, the local period of LPGs was directly measured by illuminating the fiber from its side and measuring the diffraction pattern. However, this method was not used for measuring the amplitude of the refractive index modulation of LPGs owing to the complicated diffraction pattern. ${ }^{18}$ Moreover, such a method cannot be implemented for interrogating LPG-based sensors. To the best of our knowledge, the structure of a LPG has not been measured yet. A reconstruction technique for interrogating LPGs is important for developing novel distributed fiber sensors and for improving the writing process and the performance of such gratings, as performed for FBGs. 9,10

In this paper we demonstrate what we believe to be a new method that allows us to extract the complex core-tocladding transmission spectrum of LPGs from their complex core-to-core transmission spectrum. By applying an inverse-scattering algorithm, we use the extracted coreto-cladding transmission spectrum together with the coreto-core transmission spectrum for uniquely reconstructing the grating structure. In order to uniquely reconstruct the grating, our method requires writing an additional grating, with a structure that should not be known a priori, in cascade to the interrogated grating. In general, the core-to-core transmission function of a LPG does not uniquely determine its core-to-cladding transmission function, and many possible core-to-cladding transmission functions may exist that correspond to the same coreto-core transmission function. However, we show that if the grating structure is composed of two cascaded gratings with a sufficient gap between them, there is only one core-to-cladding transmission function that corresponds to such a cascaded structure. Therefore, the a priori knowledge that the structure is composed of two cascaded gratings and that the gap is large enough enables a unique reconstruction of the interrogated grating. This result does not contradict previous conclusions, which state that, for an arbitrary LPG structure, both the coreto-core and the core-to-cladding complex transmission spectra are needed to uniquely reconstruct the grating structure. $^{14}$

In our analysis, we assume a coupling between the core mode and only one of the cladding modes. A similar model was used in Refs. 12-14 for implementing inversescattering algorithms for LPGs. The assumption of cou- 
pling to only a single cladding mode limits the spectral bandwidth that can be used to reconstruct the grating, since, when we increase bandwidth, coupling to other cladding modes may occur. Although this effect limits the maximum bandwidth that can be used, we were able to obtain a reconstruction of a LPG with a resolution of about eight times the grating period. The grating profile was reconstructed from the core-to-core transmission spectrum, calculated by using a multimode simulation of the cladding modes. In comparison, the FBG that was interrogated in Ref. 17 was measured with a spatial resolution that was equal to about 140 times the grating period.

The paper is organized as follows. In Section 2 we give the theoretical background required by our analysis. In Section 3 we discuss the conditions for a unique reconstruction of the interrogated grating. The conditions are given for three different types of the input data used in the algorithm. In Section 4 we discuss two algorithms for reconstructing the interrogated grating: the phaseretrieval algorithm and the deconvolution algorithm. In Section 5 we give numerical examples to demonstrate our reconstruction method.

\section{THEORETICAL MODEL FOR ANALYZING LONG-PERIOD GRATINGS}

In this section, we describe the mathematical model used for analyzing LPGs. In our analysis, we neglect the loss along the grating and consider the coupling between two copropagating modes: the core mode and one of the cladding modes. Under these assumptions, the propagation of the fields inside the grating can be described by the coupled-mode equations ${ }^{19}$

$$
\begin{gathered}
\frac{\mathrm{d} u_{c l}(k, z)}{\mathrm{d} z}-i k u_{c l}(k, z)=q(z) u_{c o}(k, z), \\
\frac{\mathrm{d} u_{c o}(k, z)}{\mathrm{d} z}+i k u_{c o}(k, z)=-q^{*}(z) u_{c l}(k, z),
\end{gathered}
$$

where $k$ is the wavenumber detuning defined by $k=\left(\beta_{c o}\right.$ $\left.-\beta_{c l}\right) / 2-\pi / \Lambda ; \beta_{c l}$ and $\beta_{c o}$ are the wavenumbers of the cladding and core modes, respectively; $u_{c l}(k, z)$ and $u_{c o}(k, z)$ are the slowly varying complex amplitudes of the cladding mode and the core mode, respectively; and $q(z)$ is the complex coupling coefficient of the grating. ${ }^{19}$ The actual electric fields inside the grating $e_{c o, c l}(k, z)$ are related to the fields $u_{c o, c l}(k, z)$ by the following relations ${ }^{20}$ :

$$
\begin{aligned}
& e_{c o}(k, z)=u_{c o}(k, z) \exp \left(i \frac{\beta_{c o}+\beta_{c l}}{2} z+i \frac{\pi}{\Lambda} z\right), \\
& e_{c l}(k, z)=u_{c l}(k, z) \exp \left(i \frac{\beta_{c o}+\beta_{c l}}{2} z-i \frac{\pi}{\Lambda} z\right),
\end{aligned}
$$

where $\Lambda$ is the grating period.

We assume that the grating is written in the region $[0, L]$ and consider the vectorial solution to Eqs. (1), $\mathbf{U}(k, z)=\left(u_{c l}(k, z), u_{c o}(k, z)\right)$, that fulfills the following boundary conditions, $\mathbf{U}(k, z=0)=(0, f(k)=1)$. The solution $\mathbf{U}(k, z)$ describes the propagation of the fields in the core and cladding modes when the core mode is excited at the input end of the grating. We define the core-to-cladding and core-to-core transmission functions $b(k)$ and $a(k)$, respectively, by $(b(k), a(k))=\mathbf{U}(k, z=L) / f(k)$. Since the system is linear, the transmission functions $a(k)$ and $b(k)$ do not depend on the choice of the input spectrum $f(k)$. Therefore, the transmission functions $a(k)$ and $b(k)$ are determined solely by the coupling coefficient $q(z)$. Using the theory of characteristics, as performed in Ref. 21, the functions $a(k)$ and $b(k)$ can be represented by using two time-domain functions $\alpha(\tau)$ and $\beta(\tau)$ in the following integral forms:

$$
\begin{gathered}
a(k)=\exp (-i k L)+\int_{-L}^{L} \alpha(\tau) \exp (i k \tau) \mathrm{d} \tau \\
b(k)=\int_{-L}^{L} \beta(\tau) \exp (i k \tau) \mathrm{d} \tau .
\end{gathered}
$$

The time-domain functions $\alpha(\tau)$ and $\beta(\tau)$ are equal to the Fourier transform of the functions $a(k)-\exp (-i k L)$ and $b(k)$, respectively, and are nonzero only in the time region $\tau \in[-L, L]$.

When the energy is conserved, the amplitudes of the core-to-cladding and core-to-core transmission spectra are connected by the conservation of power relation, obtained from Eqs. (1),

$$
|a(k)|^{2}+|b(k)|^{2}=1 .
$$

In characterizing LPGs, the transmission function $a(k)$ can be easily measured. When the grating is lossless, the core-to-core transmission function $a(k)$ can be used to find the amplitude of the core-to-cladding transmission function $|b(k)|$. However, there is no general relation between the phase of the function $b(k)$ and the function $a(k)$. Furthermore, in contrast to the case of FBGs, the function $a(k)$ is not necessarily a minimum phase-shift function, and therefore there is no unique relation between the phase and the amplitude of the function $a(k) .{ }^{14}$

Since Eqs. (1) are linear ordinary differential equations of $\mathbf{U}(k, z)$, the fields at the output end of the grating, $\mathbf{U}(k, z=L)$, are equal to a linear superposition of the fields at the input end of the grating, $\mathbf{U}(k, z=0)$. Thus, we can define a transfer matrix, $\mathbf{T}(k)$, that connects the fields at the input end of the grating, $z=0$, and the fields at the output end of the grating, $z=L$ :

$$
\mathbf{U}(k, z=L)=\mathbf{T}(k) \mathbf{U}(k, z=0) .
$$

Using the symmetry of Eqs. (1), it can be shown that the transfer matrix is given by ${ }^{14,22}$

$$
\mathbf{T}(k)=\left[\begin{array}{cc}
a^{*}(k) & b(k) \\
-b^{*}(k) & a(k)
\end{array}\right] .
$$

The transfer matrix of a propagation through a uniform fiber $(q(z)=0)$ with a length of $L_{f}$ is given by

$$
\mathbf{T}_{f}(k)=\left[\begin{array}{cc}
\exp \left(i k L_{f}\right) & 0 \\
0 & \exp \left(-i k L_{f}\right)
\end{array}\right] .
$$

In Section 3 of this paper, we show that a unique reconstruction of a LPG from its core-to-core transmission spec- 
trum is possible when an additional grating is written in cascade to the interrogated grating. A schematic description of the cascaded structure is given in Fig. 1. The lengths of the auxiliary and the interrogated gratings are denoted by $L_{1}$ and $L_{2}$, respectively. The transfer matrix of the whole structure can be calculated by multiplying the transfer matrices of the gratings with a free-propagation matrix,

$$
\mathbf{T}_{\mathrm{tot}}(k)=\mathbf{T}_{2}(k) \mathbf{T}_{f}(k) \mathbf{T}_{1}(k),
$$

where $\mathbf{T}_{1}(k)$ and $\mathbf{T}_{2}(k)$ are the transfer matrices of the two gratings and $\mathbf{T}_{f}(k)$ is the transfer matrix of the gap. Using Eq. (8), we calculate the core-to-core and core-tocladding transmission functions:

$$
\begin{aligned}
& a_{\text {tot }}(k)=a_{1}(k) a_{2}(k) \exp \left(-i k L_{f}\right)-b_{1}(k) b_{2}^{*}(k) \exp \left(i k L_{f}\right), \\
& b_{\text {tot }}(k)=a_{1}(k) b_{2}(k) \exp \left(-i k L_{f}\right)+b_{1}(k) a_{2}^{*}(k) \exp \left(i k L_{f}\right),
\end{aligned}
$$

where $a_{\ell}(k), b_{\ell}(k)$ are the coefficients of the transfer matrix of the $\ell$ th grating $(\ell=1,2)$, as given in Eq. (6).

When the grating is weak [i.e., $\int_{-\infty}^{\infty}|q(z)| \mathrm{d} z \ll 1$ ], the Born approximation can be used. In this case, the grating is considered a small perturbation, $q(z)=\epsilon q^{1}(z)(\epsilon \ll 1)$, and the transmission functions are given by

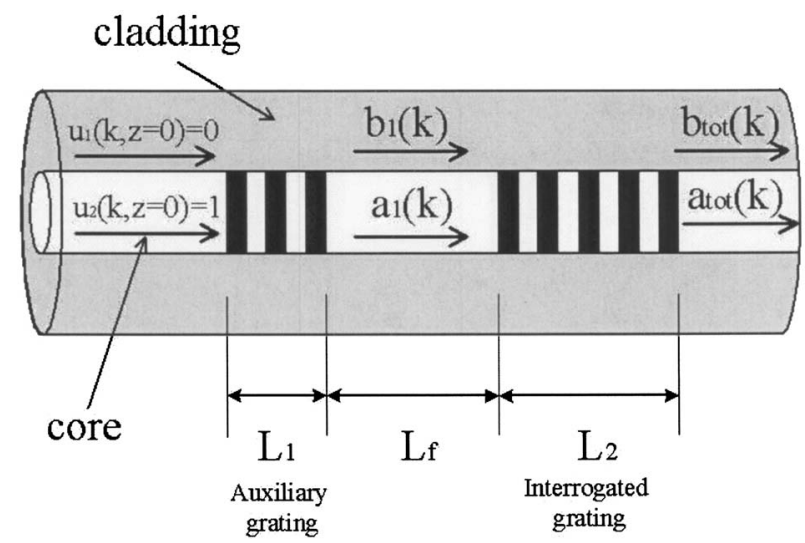

Fig. 1. Schematic description of the grating structure analyzed in the paper. Two cascaded LPGs with lengths $L_{1}$ and $L_{2}$ are separated by a gap with a length $L_{f}$. The fields of the core mode and cladding mode at the input end of the structure are denoted by $u_{2}(k, z=0)$ and $u_{1}(k, z=0)$, respectively. The core-to-core and core-to-cladding transmission functions of the first grating are denoted by $a_{1}(k)$ and $b_{1}(k)$, respectively, and the core-to-core and core-to-cladding transmission functions of the total structure are denoted by $a_{\text {tor }}(k)$ and $b_{\text {tot }}(k)$, respectively.

$$
\begin{gathered}
a(k)=\exp (-i k L)+O\left(\epsilon^{2}\right), \\
b(k)=\epsilon \frac{1}{2} \int_{-L}^{L} q^{1}\left(\frac{L-\tau}{2}\right) \exp (i k \tau) \mathrm{d} \tau+O\left(\epsilon^{3}\right) .
\end{gathered}
$$

Using Eqs. (3) and (10), we find that the coupling coefficient can be directly calculated from the function $\beta(\tau): q(\tau) \simeq 2 \beta(L-2 \tau)$.

\section{UNIQUENESS OF THE RECONSTRUCTION}

It has been shown in Refs. 13 and 14 that LPGs can be uniquely reconstructed when both the complex core-tocore and the core-to-cladding transmission functions are known. However, only the complex core-to-core transmission function of LPGs can be easily measured. Therefore, in order to reconstruct the profile of a LPG, we need to extract the complex core-to-cladding transmission function of the grating from its complex core-to-core transmission function. Generally, the reconstruction of the complex core-to-cladding transmission function is not unique, and the grating cannot be reconstructed from its core-to-core transmission function. However, in the case of a cascaded grating structure, a unique reconstruction is possible if the gap between the gratings is sufficiently long.

In this section we refer to three different cases of the reconstruction problem; in each case different input data are given. In the first case, only the complex core-to-core transmission function of the total structure, $a_{\text {tot }}(k)$, is known. In the second case, both the complex core-to-core transmission function of one of the cascaded gratings and the complex core-to-core transmission function of the total structure, $a_{\text {tot }}(k)$, are known. In the third case, we assume that the two gratings are weak and only the amplitude of the core-to-core transmission function of the total structure, $\left|a_{\text {tot }}(k)\right|$, is known. The constraints that ensure a unique reconstruction in the three cases described in this section are summarized in Table 1.

We assume in our analysis that the grating structure is lossless and, therefore, the amplitude of the function $b_{\text {tot }}(k)$ can be calculated from the amplitude of the function $a_{\text {tot }}(k)$ by using the conservation of power relation, given in Eq. (4). We note that our numerical simulation, described below, shows that even when there is a small loss along the grating structure we can still use Eq. (4) for finding a good approximation to the amplitude of the function $b_{\text {tot }}(k)$. In this case, the loss will cause only a small decrease in the amplitude of the reconstructed grating.

Since the amplitude of the function $b_{\text {tot }}(k)$ can be easily calculated by using Eq. (4), only the phase of the function $b_{\text {tot }}(k)$, denoted by $\phi_{b}(k)$, is needed to ensure a unique re-

Table 1. Summary of Reconstruction Cases and Methods

\begin{tabular}{lcccc}
\hline Case $^{a}$ & Data & Constraints & Stability against Noise & Proposed Reconstruction Methods \\
\hline 1 & $a_{\text {tot }}(k)$ & $L_{f}>2\left(L_{1}+L_{2}\right)$ & Unstable & Phase retrieval \\
2 & $a_{\text {tot }}(k)$ and $a_{1}(k)$ & $L_{f}>L_{1}+L_{2}$ & Stable if $L_{1} \ll L_{2}$ & Phase retrieval or deconvolution \\
3 & $\left|a_{\text {tot }}(k)\right|$ & $L_{f}>\max \left\{L_{1}, L_{2}\right\}$ and $\left|a_{1,2}(k)\right| \gtrsim 0.9$ & Stable if $L_{1} \ll L_{2}$ & Phase retrieval \\
\hline
\end{tabular}

${ }^{a}$ Cases 1,2 , and 3 correspond to the cases presented in Subsections 3.A-3.C, respectively. 
construction of the grating. In general, the retrieval of the phase of a spectral function from its amplitude is not unique. ${ }^{23}$ In Ref. 23 , the uniqueness of the phase-retrieval problem is studied for spectral functions whose Fourier transform has a disconnected support. We define the support of a spectral function, denoted by $D$, as the union of all the intervals in which the Fourier transform of the function is not equal to zero. When the support $D$ is a union of two nonoverlapping intervals, the phaseretrieval problem has a unique solution if the width of each of the two intervals is less than the width of the gap between them. ${ }^{23}$

The uniqueness theorem in Ref. 23 ignores trivial transformations of the reconstructed phase function $\phi_{b}(k)$, i.e., $\pm \phi_{b}(k)+\alpha k+\beta$, where $\alpha$ and $\beta$ are constants. Thus, a unique solution defines a family of solutions given by the above transformations. The trivial transformations may cause a spatial inversion of the reconstructed grating profile, $q(z) \rightarrow q(-z)^{*}$, a displacement in the position, $q(z)$ $\rightarrow q(z+\alpha)$, and a constant change in the phase, $q(z)$ $\rightarrow q(z) \exp (i \beta)$, of the grating profile. ${ }^{24}$ The last two transformations do not affect the reconstruction of the grating, and the first transformation can be overcome if the spatial order of how the two gratings were written in the fiber is known.

\section{A. Reconstruction from the Complex Core-to-Core Transmission Function of the Cascade Structure}

We consider the first case where only the complex core-tocore transmission function of the cascaded structure, $a_{\text {tot }}(k)$, is known. Following Eqs. (3) and (9), we find that the support of the Fourier transform of the function $b_{\text {tot }}(k)$ is given by

$$
\begin{aligned}
D= & {\left[-\left(L_{1}+L_{2}+L_{f}\right), L_{1}+L_{2}-L_{f}\right] \cup\left[-\left(L_{1}+L_{2}-L_{f}\right), L_{1}\right.} \\
& \left.+L_{2}+L_{f}\right],
\end{aligned}
$$

where $L_{1}$ and $L_{2}$ are the lengths of the two cascaded gratings and $L_{f}$ is the distance between them, as shown in Fig. 1. Therefore, the function $b_{\text {tot }}(k)$ fulfills the support constraint required for uniquely extracting its phase $\mathrm{p}^{23}$ if

$$
L_{f}>2\left(L_{1}+L_{2}\right) \text {. }
$$

When the condition in expression (12) is fulfilled, the phase of the core-to-cladding transmission function of the total structure, $b_{\text {tot }}(k)$, can be retrieved from its amplitude and used to reconstruct the grating structure. We note that in this reconstruction problem, and in the following problems, any increment in the gap beyond the lower bound that is required for a unique reconstruction is not necessary and does not add any information to the reconstruction problem.

\section{B. Reconstruction from the Complex Core-to-Core Transmission Functions of the Cascaded Structure and the Axillary Grating}

We consider the second case where both the complex coreto-core transmission function of the cascaded structure, $a_{\text {tot }}(k)$, and the core-to-core transmission function of the auxiliary grating $a_{1}(k)$ are known. The requirement in expression (12) ensures the uniqueness of the reconstruction of the cascaded structure from its core-to-core trans- mission function. However, when the core-to-core transmission function of one of the auxiliary gratings is also known, the uniqueness of the solution can be ensured for a narrower spatial gap between the gratings than given in expression (12). Equations (9) show that the function $a_{\text {tot }}(k)$ is composed of two elements, $a_{1}(k) a_{2}(k) \exp \left(-i k L_{f}\right)$ and $b_{1}(k) b_{2}(k)^{*} \exp \left(-i k L_{f}\right)$. According to Eqs. (3), the supports of the Fourier transform of these two elements are $\left[-\left(L_{1}+L_{2}+L_{f}\right), L_{1}+L_{2}-L_{f}\right]$ and $\left[-\left(L_{1}+L_{2}-L_{f}\right), L_{1}+L_{2}+L_{f}\right]$, respectively. The supports of the Fourier transform of the two elements do not overlap if the following condition is met:

$$
L_{f}>\left(L_{1}+L_{2}\right) \text {. }
$$

In this case, we can extract the functions $a_{1}(k) a_{2}(k)$ and $b_{1}(k) b_{2}^{*}(k)$ from the function $a_{\mathrm{tot}}(k)$. Since one of the functions $a_{1}(k)$ and $a_{2}(k)$ is also known, the other function can be computed from the product $a_{1}(k) a_{2}(k)$. Then, the preservation of power relation can be used for calculating the amplitude functions $\left|b_{1}(k)\right|$ and $\left|b_{2}(k)\right|$. The functions $\left|b_{1}(k)\right|,\left|b_{2}(k)\right|$ and the product $b_{1}(k) b_{2}^{*}(k)$ can be used to uniquely determine the functions $b_{1}(k)$ and $b_{2}(k)$ by defining

$$
\hat{b}(k)=b_{1}(k)+b_{2}(k) \exp (i k \hat{L}),
$$

where $\hat{L}$ is an arbitrary constant that fulfills the separation condition $\hat{L}>L_{1}+L_{2}$. The intensity and, hence, the amplitude of the function $\hat{b}(k)$ can be calculated from the known functions $\left|b_{1}(k)\right|,\left|b_{2}(k)\right|$ and the product $b_{1}(k) b_{2}^{*}(k)$. Since the function $\hat{b}(k)$ fulfills the separation condition, it can be determined uniquely by its intensity. Since $\hat{L}$ fulfills the separation condition, the functions $b_{1}(k)$ and $b_{2}(k)$ can be uniquely calculated from the extracted function $\hat{b}(k)$.

\section{Reconstruction from the Core-to-Core Transmission Intensity of the Cascaded Structure}

We consider the third case where only the core-to-core transmission intensity of the cascaded structure is known. When the two gratings are weak (the minimum transmissivity of the core-to-core mode along the grating $\lesssim 20 \%$ ), the Born approximation can be used, and the grating can be reconstructed from only the amplitude of the core-to-core transmission function. In this case, the transmission function $b_{\text {tot }}(k)$ can be approximated by

$$
b_{\text {tot }}(k) \simeq b_{2}(k) \exp \left[-i k\left(L_{f}+L_{1}\right)\right]+b_{1}(k) \exp \left[i k\left(L_{f}+L_{2}\right)\right] \text {, }
$$

and the support of the Fourier transform of the function $b_{\text {tot }}(k)$ is given by

$$
\begin{aligned}
D= & {\left[-L_{1}-L_{2}-L_{f}, L_{1}-L_{2}-L_{f}\right] \cup\left[L_{1}-L_{2}+L_{f}, L_{1}\right.} \\
& \left.+L_{2}+L_{f}\right] .
\end{aligned}
$$

Therefore, the function $b_{\text {tot }}(k)$ can be uniquely determined from its amplitude if

$$
L_{f}>\max \left(L_{1}, L_{2}\right) .
$$

Once the function $b_{\text {tot }}(k)$ is calculated, the grating can be reconstructed by using Eqs. (10), without the use of 
inverse-scattering algorithms. Since the function $b_{\text {tot }}(k)$ is uniquely calculated from the amplitude of the core-to-core transmission function of the total structure, $a_{\text {tot }}(k)$, only the function $\left|a_{\text {tot }}(k)\right|$ is required for reconstructing the grating. It can be shown that, in this case, additional information on the core-to-core transmission function of one of the cascaded gratings does not reduce the separation constraint given in expression (17).

\section{METHODS FOR EXTRACTING THE CORE-TO-CLADDING TRANSMISSION FUNCTION}

In this section, we describe two methods for reconstructing the complex core-to-cladding transmission function of a cascaded grating structure from its core-to-core transmission function. The first method is based on a phaseretrieval algorithm. The method requires knowledge of the core-to-core transmission function of the total structure; however, the phase-retrieval method allows us to obtain better results when the core-to-core transmission function of the auxiliary grating is also known. The second method is based on a direct deconvolution operation. This method requires knowledge of the core-to-core transmission functions of both the auxiliary grating and the total structure and can be used for the reconstruction problem of Subsection 3.B.

\section{A. Phase-Retrieval Algorithm}

Phase-retrieval algorithms may be classified into three categories: optimization algorithms ${ }^{25}$ stochastic algorithms, ${ }^{26,27}$ and iterative algorithms. ${ }^{28,29}$ Optimization algorithms are based on minimizing an error functional defined according to the characteristics of the specific phaseretrieval problem in study. In our problem the Fourier transform of the spectrum should be zero for a given time interval, and the error functional is defined as the energy of the error integrated over the interval. The global minimum of the error functional gives the solution to the phase-retrieval problem. The main drawback of optimization algorithms is that they may converge to local minima of the error functional, which do not correspond to the solution of the phase-retrieval problem. ${ }^{25}$ Stochastic algorithms such as the genetic algorithm ${ }^{26}$ or the simulated annealing algorithm ${ }^{27}$ are more capable of overcoming the problem of local minima. However, the computational complexity of these algorithms is high, and, therefore, the computational time needed for obtaining the results may be very long. ${ }^{26,27}$ The third type of phase-retrieval algorithms is based on an iterative scheme, which imposes constraints in both time and frequency domains. One well-known iterative algorithm is the hybrid input-output (HIO) algorithm. ${ }^{28}$ The advantage of the HIO algorithm is that it allows one to overcome the problem of local minima more efficiently than stochastic algorithms. The convergence properties of the $\mathrm{HIO}$ algorithm are studied in Refs. 29 and 30.

In our work we use the HIO algorithm for all the cases discussed in Section 3. We define the function $\beta_{\text {tot }}(\tau)$ as the Fourier transform of the core-to-cladding transmission function, $b_{\text {tot }}(k)$. Given the amplitude function $\left|b_{\text {tot }}(k)\right|$, we require that the output function of the HIO algorithm, denoted by $\beta_{\text {sol }}(\tau)$, fulfill the constraints

$$
\begin{gathered}
\beta_{\text {sol }}(\tau)=0, \quad \text { for } \tau \notin D, \\
\left|b_{\text {sol }}(k)\right|=\left|b_{\text {tot }}(k)\right|,
\end{gathered}
$$

where $b_{\mathrm{sol}}(k)$ is the inverse Fourier transform of the function $\beta_{\text {sol }}(\tau)$, and $D$ is the support of the function $\beta_{\text {tot }}(\tau)$, given in Eq. (11). According to the uniqueness theorem in Ref. 23 , the function $\beta_{\text {sol }}(\tau)$, which fulfills the constraints in Eqs. (18) and (19), is equal to the desired solution, $\beta_{\text {tot }}(\tau)$.

We now describe the HIO algorithm used in our work. We define $\beta_{n}^{\text {in }}(\tau)$ and $\beta_{n}^{\text {out }}(\tau)$ as the input and the output functions of the $n$th iteration of the HIO algorithm and $b_{n}^{\text {in }}(k)$ and $b_{n}^{\text {out }}(k)$ as their corresponding inverse Fourier transforms. The HIO algorithm can be summarized by the following iterative relation ${ }^{28}$ :

$$
\begin{gathered}
b_{n}^{\text {out }}(k)=\frac{b_{n}^{\text {in }}(k)}{\left|b_{n}^{\text {in }}(k)\right|}\left|b_{\text {tot }}(k)\right|, \\
\beta_{n+1}^{\text {in }}(\tau)=\left\{\begin{array}{ll}
\beta_{n}^{\text {out }}(\tau), & \tau \in D \\
\beta_{n}^{\text {in }}(\tau)-\gamma \beta_{n}^{\text {out }}(\tau), & \tau \notin D
\end{array},\right.
\end{gathered}
$$

where $\gamma$ is a constant feedback parameter. The starting guess for the algorithm, $\beta_{1}^{\text {in }}(\tau)$, can be chosen arbitrarily. Equations (20) show that if the algorithm converges in the sense that $\lim _{n \rightarrow \infty}\left|\beta_{n+1}^{i n}(\tau)-\beta_{n}^{i n}(\tau)\right|=0$, then the output of the algorithm would fulfill the condition $\lim _{n \rightarrow \infty} \beta_{n}^{\text {out }}(\tau)$ $=0$ for $\tau \notin D$. Therefore, if the algorithm converges, the output of the algorithm, $\beta_{\mathrm{sol}}(\tau)$, fulfills the constraints in Eq. (18), and its solution is the solution to the phaseretrieval problem. We define the error functional $E$ as

$$
E=\int_{\tau \notin D}\left|\beta_{\mathrm{sol}}(\tau)\right|^{2} \mathrm{~d} \tau .
$$

The error functional $E$ is used as a practical criterion for the convergence of the algorithm; the algorithm is said to have converged when the error $E$ is smaller than a desired value.

The properties of the HIO algorithm are described in Refs. 29 and 30. In those references, the authors distinguish between two parts of the solution of the algorithm: the converging part and the emerging part. The converging part of the HIO algorithm allows the algorithm to converge to functions that have a local minimum for the error functional $E$, and the emerging part causes the algorithm to emerge from a local minimum that is not the solution. However, it is not known how many iterations are required for the convergence and what is the optimal value of $\gamma$. Therefore, the choice of the number of iterations and of the value of $\gamma$ is made empirically. ${ }^{28}$

When the function $\left|b_{\text {tot }}(k)\right|$ is obtained from a measurement, it contains noise, and, therefore, there may be no solution to the phase-retrieval problem. In this case, we define our solution as the function $\beta_{\text {sol }}(\tau)$ that fulfills Eq. (19) and minimizes the error functional $E$, defined in Eq. (21). However, the HIO algorithm can converge only to a state where the error functional $E$ is zero. ${ }^{30}$ Therefore, in 
the case of noisy data, the HIO algorithm may not converge, and the solution may alternate between different local minima of the error functional. ${ }^{29}$ To overcome this problem, a variation of the HIO algorithm was developed in Ref. 29. This method, called the separated hybrid input-output (SHIO) algorithm, separates the converging part and the emerging part of the HIO algorithm. Therefore, the SHIO algorithm allows convergence to a local minimum state that is not the solution. Once a convergence is obtained, the emerging part is used to locate a new local minimum state. The local minimum state with the lowest error functional is considered the solution of the algorithm. The SHIO algorithm is summarized in Appendix A.

The SHIO and HIO algorithms, described in this subsection, are used to solve the reconstruction problem considered in Subsection 3.A, where the core-to-core transmission function of the cascaded structure $a_{\text {tot }}(k)$ is known. The number of iterations required by the SHIO algorithm for converging to the solution of the phaseretrieval problem depends on the function $\beta_{\text {tot }}(\tau)$. Furthermore, the sensitivity of the solution to noise also depends on the function that should be reconstructed. We found that when the support $D$ is composed of two disconnected intervals that have significantly different lengths the algorithm is less sensitive to noise and its convergence becomes faster. However, in the reconstruction problem considered in Subsection 3.A, the support of the function $\beta_{\text {tot }}(\tau)$ is generally composed of two equal intervals with the same length, $2\left(L_{1}+L_{2}\right)$. In this case, the reconstruction using the SHIO algorithm may have a high sensitivity to noise and may slowly converge.

The sensitivity to noise of the phase-retrieval algorithms can be overcome in the reconstruction cases considered in Subsections 3.B and 3.C. In the case of Subsection 3.B, where the core-to-core transmission functions of the auxiliary grating and total structure are known, the SHIO and HIO algorithms are used to extract the phase of the function $\hat{b}(k)$, defined in Eq. (14), instead of the phase of the function $b_{\text {tot }}(k)$. Since the support of the function $\hat{b}(k)$ is composed of two intervals with lengths of $2 L_{1}$ and $2 L_{2}$, the sensitivity of the SHIO algorithm to noise can be significantly reduced if the length of the auxiliary grating is considerably shorter than the length of the interrogated grating. In the case of Subsection 3.C, where the gratings are weakly coupling and the Born approximation can be used, the support of the function $\beta_{\text {tot }}(\tau)$ is also composed of two intervals with lengths of $2 L_{1}$ and $2 L_{2}$. Therefore, in this case as well, the performance of the SHIO algorithm can be enhanced by choosing a short auxiliary grating.

\section{B. Deconvolution Method}

The deconvolution method is based on a direct calculation of the transmission spectrum of the interrogated grating from the core-to-core transmission spectra of the auxiliary grating and of the total structure. Similarly to the case of the phase-retrieval algorithm, we have found out that in order to obtain stable results in the presence of noise the auxiliary grating should be considerably shorter than the interrogated grating.
As shown below, the minimum gap between the gratings, required by the deconvolution method, is equal to the sum of the grating lengths $L_{1}+L_{2}$, as obtained in expression (13). When the condition in expression (13) is fulfilled, the functions $a_{2}(k),\left|b_{1}(k)\right|^{2}$, and $b_{1}(k) b_{2}^{*}(k)$ can be calculated from the core-to-core transmission functions of both the auxiliary grating and the total structure, $a_{1}(k)$ and $a_{\text {tot }}(k)$, as explained in Section 3. In this subsection, we will show how we can extract the function $b_{2}^{*}(k)$ from the product $b_{1}(k) b_{2}^{*}(k)$ by using the known intensity function $\left|b_{1}(k)\right|^{2}$.

The deconvolution method requires a discrete representation of the core-to-cladding transmission functions of the two gratings $b_{\ell}(k)(\ell=1,2)$. When the functions $b_{\ell}(k)$ are uniformly sampled with a sampling period of $\Delta k$ $>\pi / \max \left\{L_{\ell}\right\}$, the Fourier transform of the functions $b_{\ell}(k)$ can be calculated for $|\tau|<\pi / \Delta k$ by using

$$
\beta_{\ell}(\tau)=\frac{\Delta k}{2 \pi} \sum_{m=-\infty}^{\infty} b_{\ell}\left(k_{m}\right) \exp \left(-i k_{m} \tau\right),
$$

where $k_{m}=m \Delta k$ and $m$ is an integer number. We note that the restriction on the sampling period $\Delta k$ ensures that there is no error due to the aliasing effect in the calculation. $^{31}$ In practice, the core-to-cladding transmission function can be obtained only over a finite bandwidth, and, therefore, we approximate the functions $\beta_{\ell}(\tau)$ by

$$
\beta_{\ell}\left(\tau_{n}\right) \cong \frac{\Delta k}{2 \pi} \sum_{m=-N}^{N} b_{\ell}\left(k_{m}\right) \exp \left(-i k_{m} \tau_{n}\right),
$$

where $\tau_{n}=n \Delta \tau, \Delta \tau=2 \pi /[(2 N+1) \Delta k]$, and $n=-N, \ldots, N$. We use the inverse discrete Fourier transform on both sides of expression (23) and obtain

$$
b_{\ell}\left(k_{m}\right) \cong \Delta \tau \sum_{n=-N_{\ell}}^{N_{\ell}} \beta_{\ell}\left(\tau_{n}\right) \exp \left(i k_{m} \tau_{n}\right),
$$

where $N_{\ell}=L_{\ell} / \Delta \tau$. We note that if the function $\beta_{\ell}(\tau)$ has an abrupt discontinuity, caused by a discontinuity in the grating profile, then the support region of the function may become wider than $\left[-L_{\ell}, L_{\ell}\right]$ owing to the Gibbs phenomenon. In this case the lengths of the gratings $L_{\ell}$ should be defined slightly longer than their actual lengths.

The $z$ transform of the functions $\beta_{\ell}\left(\tau_{n}\right)$ is defined as

$$
b_{\ell}(z)=\sum_{n=-N_{\ell}}^{N_{\ell}} \beta_{\ell}\left(\tau_{n}\right) z^{-n} \text {. }
$$

Since the function $b_{\ell}(z)$ is a polynomial function of the variable $z$, it can be represented by the product

$$
b_{\ell}(z)=\beta_{\ell}\left(\tau_{N_{\ell}}\right) z^{-N_{\ell}} \prod_{j=1}^{2 N_{\ell}}\left(z-\zeta_{\ell}^{j}\right),
$$

where $\left\{\zeta_{\ell}^{j}\right\}$ are the complex roots of the functions $b_{\ell}(z)(\ell$ $=1$, 2). Using the functions $\left|b_{1}\left(k_{m}\right)\right|^{2},\left|b_{2}\left(k_{m}\right)\right|^{2}$, and $b_{1}\left(k_{m}\right) b_{2}^{*}\left(k_{m}\right)$, we calculate the $z$-transformed functions $b_{1}(z) b_{1}^{*}\left(1 / z^{*}\right), b_{2}(z) b_{2}^{*}\left(1 / z^{*}\right)$, and $b_{1}(z) b_{2}^{*}\left(1 / z^{*}\right)$, which can be represented by ${ }^{31}$ 


$$
\begin{gathered}
b_{1}(z) b_{1}^{*}\left(1 / z^{*}\right)=\left|\beta_{1}\left(\tau_{N_{1}}\right)\right|^{2} \prod_{j=1}^{2 N_{1}}\left(z-\xi_{1}^{j}\right)\left(z^{-1}-\left\{\zeta_{1}^{j}\right\}^{*}\right), \\
b_{2}(z) b_{2}^{*}\left(1 / z^{*}\right)=\left|\beta_{2}\left(\tau_{N_{2}}\right)\right|^{2} \prod_{j=1}^{2 N_{2}}\left(z-\zeta_{2}^{j}\right)\left(z^{-1}-\left\{\zeta_{2}^{j}\right\}^{*}\right), \\
b_{1}(z) b_{2}^{*}\left(1 / z^{*}\right)=\beta_{1}\left(\tau_{N_{2}}\right)\left\{\beta_{2}\left(\tau_{N_{1}}\right)\right\}^{*} \prod_{j=1}^{N_{1}}\left(z-\zeta_{1}^{j}\right) \prod_{i=1}^{N_{2}}\left(z^{-1}-\left\{\zeta_{2}^{i}\right\}^{*}\right) .
\end{gathered}
$$

The zeros of the function $b_{1}(z) b_{1}^{*}\left(1 / z^{*}\right)$ are obtained in a conjugate reciprocal pair: $\left\{\zeta_{1}^{j}, 1 /\left\{\zeta_{1}^{j}\right\}^{*}\right\}$. When only the function $b_{1}(z) b_{1}^{*}\left(1 / z^{*}\right)$ is known, there is no indication of whether $\zeta_{1}^{j}$ or $1 /\left\{\zeta_{1}^{j}\right\}^{*}$ is the zero of the function $b_{1}(z)$. In this case, there are $2^{N_{1}}$ different functions $b_{1}(z)$ corresponding to the same intensity function $\left|b_{1}\left(k_{m}\right)\right|^{2}$. In order for us to obtain a unique solution, the functions $b_{1}(z)$ and $b_{2}(z)$ should not share a common zero. In this case, the comparison of the zeros of the function $b_{1}(z) b_{2}^{*}\left(1 / z^{*}\right)$ and the function $b_{1}(z) b_{1}^{*}\left(1 / z^{*}\right)$ gives the zeros of the function $b_{1}(z)$. Therefore, the functions $\left|b_{1}\left(k_{m}\right)\right|^{2},\left|b_{2}\left(k_{m}\right)\right|^{2}$, and $b_{1}\left(k_{m}\right) b_{2}^{*}\left(k_{m}\right)$ determine uniquely both functions $b_{1}\left(k_{m}\right)$ and $b_{2}\left(k_{m}\right)$.

The calculation of the zeros of the functions $b_{1}(z) b_{1}^{*}\left(1 / z^{*}\right)$ and $b_{1}(z) b_{2}^{*}\left(1 / z^{*}\right)$ requires finding the zeros of polynomial functions of the order of $2 N_{1}$ and $N_{1}+N_{2}$, respectively. Such a calculation is a numerical operation with a very high complexity that strongly increases with the number of roots of the function. The calculation of the zeros of the function $b_{1}(z) b_{1}^{*}\left(1 / z^{*}\right)$ can be simplified by choosing an auxiliary grating with a short length and a small number of sampled points, $N_{1}$. However, such a restriction cannot be made on the length of the interrogated grating. Therefore, we used a different method to find the zeros of the function $b_{1}(z)$ without calculating the roots of the function $b_{1}(z) b_{2}^{*}\left(1 / z^{*}\right)$. Given a pair of zeros $\zeta_{1}^{j}$ and $1 /\left\{\zeta_{1}^{j}\right\}^{*}$, we define the following functions:

$$
\begin{gathered}
f_{j}\left(k_{m}\right)=\frac{b_{1}\left(k_{m}\right) b_{2}^{*}\left(k_{m}\right)}{\exp \left(-i k_{m} \tau_{n}\right)-\zeta_{1}^{j}}, \\
g_{j}\left(k_{m}\right)=\frac{b_{1}\left(k_{m}\right) b_{2}^{*}\left(k_{m}\right)}{1-\exp \left(-i k_{m} \tau_{n}\right)\left\{\zeta_{1}^{j}\right\}^{*}} .
\end{gathered}
$$

Only in one of the functions defined in Eqs. (28) is the added pole in the denominator canceled out by a zero in the numerators. Since the numerators of the functions $f_{j}\left(k_{m}\right)$ and $g_{j}\left(k_{m}\right)$ do not contain poles, the Fourier transform of only one of the functions $f_{j}\left(k_{m}\right)$ and $g_{j}\left(k_{m}\right)$ should have a finite support of $N_{1}+N_{2}$ points for a specific value of $j$. Therefore, the zeros of the function $b_{1}(z)$ can be determined by checking the support region of the functions $f_{j}\left(k_{m}\right)$ and $g_{j}\left(k_{m}\right)$. After all the zeros of the function $b_{1}(z)$ are determined, we can calculate the discrete-time function $b_{1}\left(k_{m}\right)$ and use it to obtain the function $b_{2}\left(k_{m}\right)$ from the known product $b_{1}(k) b_{2}^{*}(k)$.
When the data are accurate, the Fourier transform of one of the functions $f_{j}\left(k_{m}\right)$ and $g_{j}\left(k_{m}\right)$ should have a finite support of $N_{1}+N_{2}$ points. When the core-to-core transmission function contains errors due to numerical inaccuracies and/or due to noise added in experiments, the zeros of the function $b_{1}(z)$ may not coincide with the zeros of the function $b_{1}(z) b_{2}^{*}\left(1 / z^{*}\right)$. In this case, the Fourier transform of both functions $f_{j}\left(k_{m}\right)$ and $g_{j}\left(k_{m}\right)$, defined in Eqs. (28), may have an infinite support, and we determine the zeros of the function $b_{1}(z)$ according to the function with the Fourier transform that has the smallest energy outside its theoretical support. An alternative method to find the zeros of the function $b_{1}(z)$ is to calculate the $2^{N_{1}}$ combinations of the function $b_{1}(z)$ from the function $b_{1}(z) b_{1}^{*}\left(1 / z^{*}\right)$. Then, the function $b_{2}\left(k_{m}\right)$ is calculated for each combination. In the case of error-free data, the Fourier transform of the function $b_{2}\left(k_{m}\right)$ should have a support of $N_{2}$ points. When the data contain errors, the optimal solution is chosen according to the function $b_{2}\left(k_{m}\right)$ with a Fourier transform that has the smallest energy outside of its theoretical support. Since the length of the auxiliary grating can be chosen to be significantly shorter than the length of the interrogated grating, the number of possible combinations, $2^{N_{1}}$, may be chosen to be small.

The extracted function $b_{1}(z)$ is used to calculate the function $b_{2}(z)$ from the known function $b_{1}(z) b_{2}^{*}\left(1 / z^{*}\right)$. When the data are noisy, the extracted zeros of the function $b_{1}(z)$ do not exactly coincide with the zeros of the function, $b_{1}(z) b_{2}^{*}\left(1 / z^{*}\right)$, and, therefore, the calculation of the function $b_{2}(z)$ becomes inaccurate. The error in the calculation of the function $b_{2}(z)$ increases when the number of noncoinciding zeros increases. Therefore, in order to minimize the error, the number of zeros of the function $b_{1}(z)$ should be as small as possible. This requirement is fulfilled by choosing the length of the auxiliary grating to be short enough, so it would contain only few sampled points. When the function $b_{1}(z)$ has zeros located near the unit circle, the error in calculating the function $b_{2}\left(k_{m}\right)$ significantly increases in frequencies where the function $b_{1}\left(k_{m}\right)$ is close to zero. Therefore, we also need to choose an auxiliary grating with a transmission function $b_{1}\left(k_{m}\right)$ that does not become too small across the measured bandwidth.

\section{NUMERICAL EXAMPLES}

In this section, we demonstrate our technique for reconstructing LPGs from their core-to-core transmission spectra for the three reconstruction cases described in Section 3. In the first example, we demonstrate the reconstruction of a cascaded structure from its complex core-tocore transmission function as described in Subsection 3.A. In the following two examples, we demonstrate the reconstruction of a cascaded structure from the core-to-core transmission functions of both the cascaded structure and the auxiliary grating, as described in Subsection 3.B. In the last example we demonstrate the reconstruction of a cascaded structure from its intensity core-to-core transmission function as described in Subsection 3.C. The reconstruction using the phase-retrieval method is 


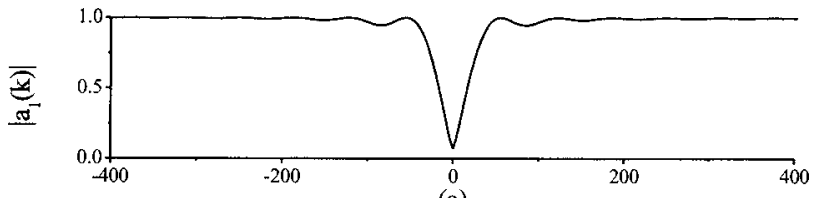

(a)

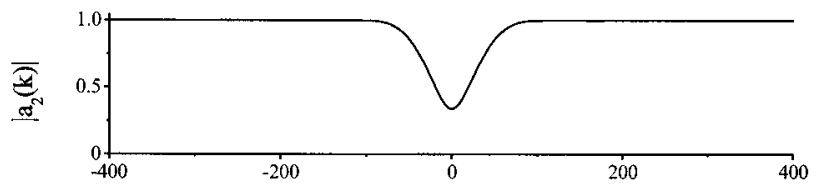

(b)

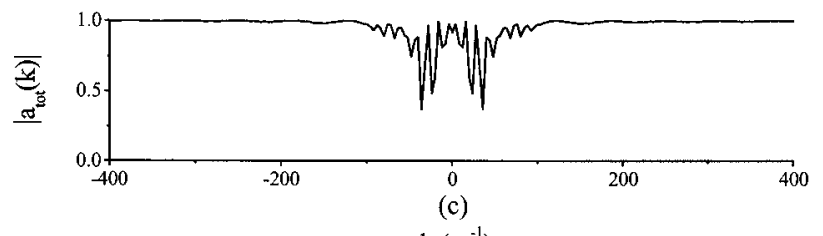

$\mathrm{k}\left(\mathrm{m}^{-1}\right)$

Fig. 2. (a) Core-to-core transmission function amplitudes of the uniform grating, (b) Gaussian grating, (c) and the total structure given in Eq. (29). The transmission spectra were calculated with a wavenumber resolution of $\Delta k=4 \mathrm{~m}^{-1}$ and a bandwidth of $4000 \mathrm{~m}^{-1}$ by using the transfer-matrix method given in Ref. 19 .

demonstrated in all the examples. The reconstruction using the deconvolution method is demonstrated for the second and the third examples.

When the phase-retrieval method was used, we first applied the HIO algorithm for a 1000 iterations to obtain a good initial input for the SHIO algorithm. The iteration of the HIO algorithm with the lowest error functional $E$ was used as the input to the SHIO algorithm, which was used for 30 iterations. The use of the HIO algorithm before the use of the SHIO algorithm allowed a faster convergence of the SHIO algorithm. ${ }^{29}$ We chose the feedback parameter to be $\gamma=0.5$ in both HIO and SHIO algorithms. In all the examples, a Hanning window was used to reduce errors in the reconstructed profile due to the Gibbs effect. ${ }^{31}$ In the case of the HIO and SHIO algorithms, we multiplied the input function of the algorithm by a Hanning window. In the case of the deconvolution method, we first calculated the core-to-cladding transmission function of the interrogated grating $b_{2}(k)$ and afterward multiplied it by the Hanning window. The Hanning window has also reduced the reconstruction error by diminishing the noise components in the high frequencies of the transmission spectrum, which were amplified by the extraction method. We chose the bandwidth of the transmission spectrum, used in the calculations, by requiring that the amplitude of the core-to-core transmission spectrum of the total structure approach 1 at the edges of the bandwidth.

In the first example, we demonstrate the reconstruction method described in Subsection 3.A. The method gave an accurate result for each grating structure we analyzed as long as the gap length between the two gratings fulfilled the condition described in expression (12). In the example, we reconstructed a structure of a uniform grating and a Gaussian grating with the same length from the complex core-to-core transmission spectrum of the total structure. The coupling coefficient of the structure is given by

$$
q(z)=\left\{\begin{array}{ll}
30 \mathrm{~m}^{-1}, & 0<z<0.05 \\
45 \exp \left[-4000(z-0.275)^{2}\right] \mathrm{m}^{-1}, & 0.25<z<0.3
\end{array} .\right.
$$

The uniform grating had approximately $100 \%$ maximum coupling between the two modes, and the Gaussian grating had about $80 \%$ maximum coupling between the two modes. The complex core-to-core transmission spectrum was calculated with a wavenumber resolution of $\Delta k$ $=4 \mathrm{~m}^{-1}$ and a wavenumber bandwidth of $4000 \mathrm{~m}^{-1}$. The calculation was performed by using the transfer-matrix method, described in Ref. 19, to solve Eqs. (1). Figure 2 shows the amplitudes of the core-to-core transmission function of the uniform grating $\left(\left|a_{1}(k)\right|\right)$, the Gaussian grating $\left(\left|a_{2}(k)\right|\right)$, and the total cascaded structure $\left(\left|a_{\mathrm{tot}}(k)\right|\right)$. The grating was reconstructed from the complex function $a_{\text {tot }}(k)$, whose amplitude is shown in Fig.

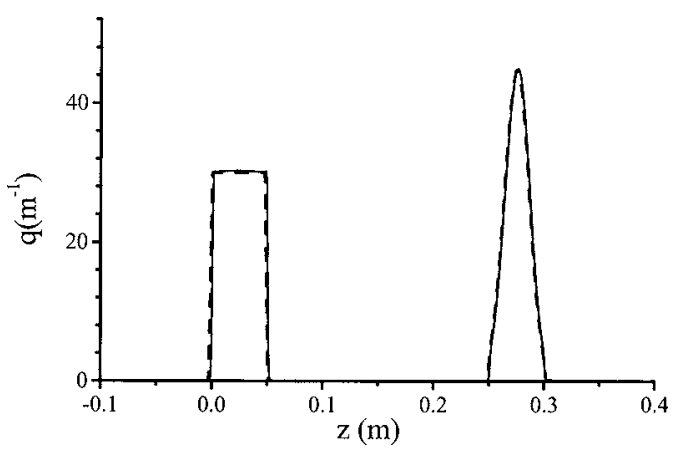

Fig. 3. Reconstruction of a cascaded structure of uniform and Gaussian gratings, given in Eq. (29), from the complex core-tocore transmission spectrum of the structure, shown in Fig. 2(c). The reconstruction was performed by using the phase-retrieval method (solid curves) and is compared with the exact grating profile (dashed curves).

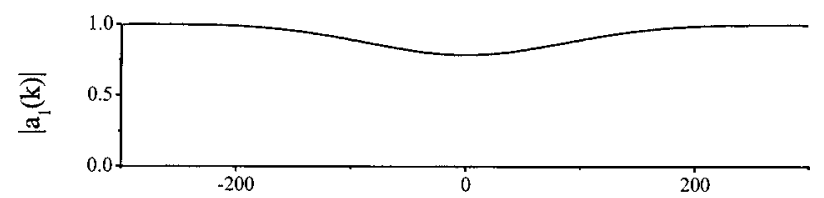

(a)
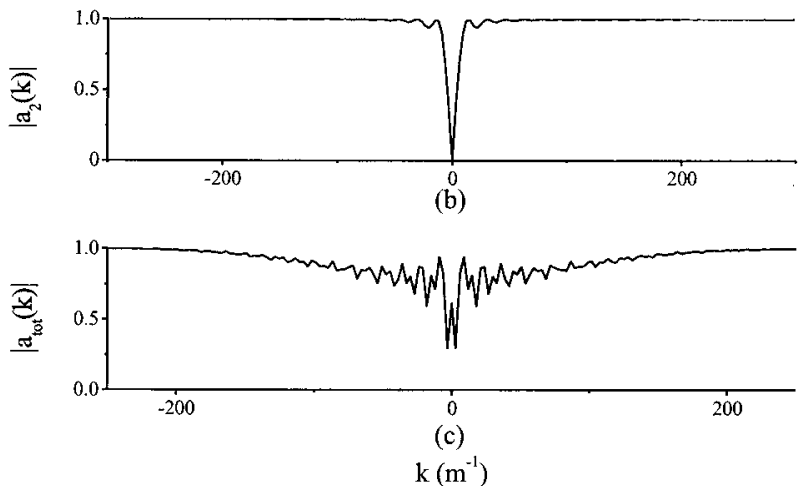

Fig. 4. (a) Core-to-core transmission function amplitudes of the short (auxiliary) grating, (b) the long (interrogated) grating, (c) and the total structure given in Eq. (30). The transmission spectra were calculated with a wavenumber resolution of $\Delta k=3 \mathrm{~m}^{-1}$ and a bandwidth of $600 \mathrm{~m}^{-1}$ by using the transfer-matrix method given in Ref. 19. 


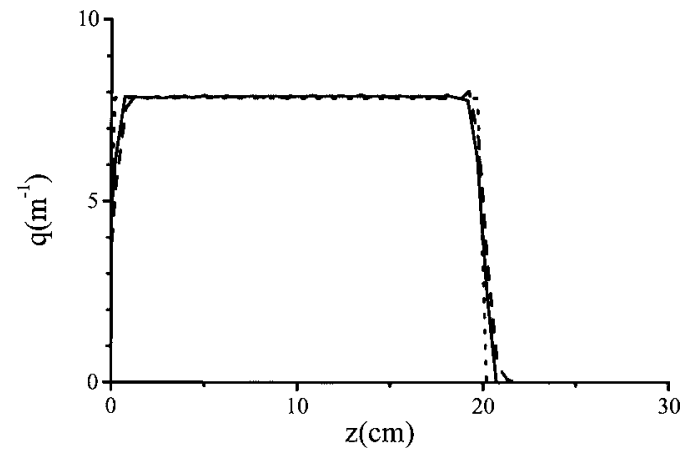

Fig. 5. Reconstruction of the long (interrogated) uniform grating in the structure described in Eq. (30) from the complex coreto-core transmission spectra shown in Figs. 4(a) and 4(c). The reconstruction was performed by using the phase-retrieval method (solid curve) and the deconvolution method (dashed curve). The reconstruction is compared with the exact grating profile (dotted curve).

2(c), by using the phase-retrieval method. The reconstruction is unique, since the gap between the gratings fulfills the condition in expression (12). Figure 3 shows the reconstructed grating profiles (solid curves) compared with the exact grating profiles (dashed curves). The figure shows that an excellent reconstruction of the two gratings was obtained. We note that the choice of the grating profiles was arbitrary and that our method was not limited by the particular grating structure. However, when noise was added to the transmission spectrum, the reconstruction became unstable.

In the second example, we demonstrate the reconstruction method described in Subsection 3.B. We did not observe a dependence of the reconstruction quality on the specific grating profiles that were chosen as long as the length of the auxiliary grating was significantly shorter than the length of the interrogated grating. In the example, we have analyzed a structure of an interrogated uniform grating with a coupling efficiency of about $100 \%$ and a length of $20 \mathrm{~cm}$ and a short auxiliary Gaussian grating with a coupling efficiency of about $39 \%$ and a length of $2 \mathrm{~cm}$. The coupling coefficient of the total structure is given by

$$
q(z)= \begin{cases}7.85 \mathrm{~m}^{-1}, & 0<z<0.2 \mathrm{~m} \\ 310 \exp \left[-5 \times 10^{4}(z-0.45)^{2}\right] \mathrm{m}^{-1}, & 0.44 \mathrm{~m}<z<0.46 \mathrm{~m} \\ 0 & \text { elsewhere }\end{cases}
$$

The gap between the gratings fulfills the condition in expression (13), and, therefore, the reconstruction of the interrogated grating structure is unique. The complex coreto-core transmission functions of the short grating and the total structure were calculated with a wavenumber resolution of $\Delta k=3 \mathrm{~m}^{-1}$ and a wavenumber bandwidth of $600 \mathrm{~m}^{-1}$ by using the same method as in the first example. Figure 4 shows the core-to-core transmissionfunction amplitudes of the auxiliary grating $\left(\left|a_{1}(k)\right|\right)$, the interrogated grating $\left(\left|a_{2}(k)\right|\right)$, and the total structure $\left(\left|a_{\text {tot }}(k)\right|\right)$. The input data for the reconstruction were the complex core-to-core transmission spectra of the auxiliary grating and total grating structure, whose amplitudes are shown in Figs. 4(a) and 4(c). We used the phase-retrieval method and the deconvolution method to reconstruct the interrogated grating profile. Figure 5 shows the reconstruction of the interrogated grating by using the phaseretrieval method (solid curve) and the deconvolution method (dashed curve); the reconstruction results are compared with the accurate coupling coefficient (dotted curve). The figure shows that both methods were able to accurately reconstruct the interrogated grating structure. We note that since the length of the auxiliary grating is short, it contains only about three points, and therefore we did not include its reconstruction in the figure.

In the third example, we added noise to the transmission functions that were used in the previous example and reconstructed the grating as in the previous example. We added to each calculated point in the core-to-core transmission spectra of the auxiliary and the total grating structure a random variable, which represented the noise in the experiment. The random variables were inde- pendent and had a Gaussian distribution with a zero mean and a standard deviation of $5 \times 10^{-3}$. Figure 6 shows the reconstruction of the interrogated grating by using the phase-retrieval method (solid curve) and the deconvolution method (dashed curve). The reconstruction results are compared with the accurate coupling coefficient (dotted curve). The figure shows that a good reconstruction is obtained by both methods. The stability of our method against noise is a result of the use of a short auxiliary grating.

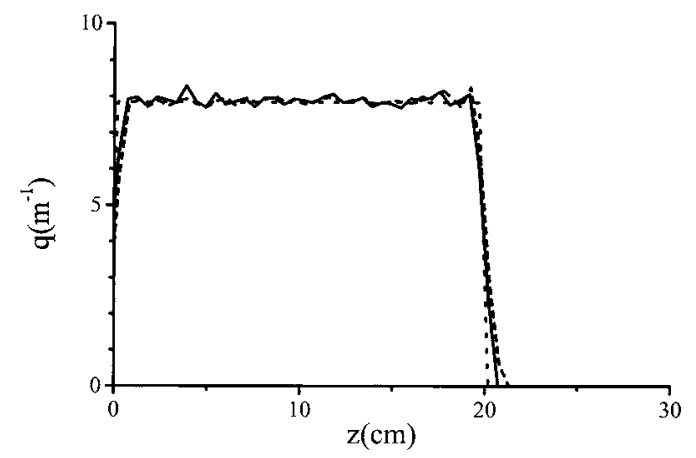

Fig. 6. Reconstruction of the long (interrogated) uniform grating in the structure given in Eq. (30) from the noisy complex coreto-core transmission spectra of the auxiliary grating and the total structure. The noise was represented by independent random variables that were added to the transmission spectra of the grating. The random variables had a Gaussian distribution with a zero mean and a standard deviation of $5 \times 10^{-3}$. The reconstruction was performed by using the phase-retrieval method (solid curve) and the deconvolution method (dashed curve). The reconstruction is compared with the exact grating profile (dotted curve). 


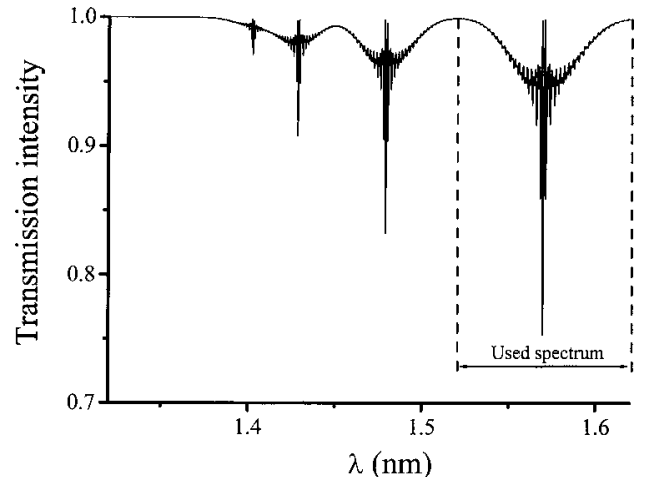

Fig. 7. Core-to-core transmission intensity of two weak gratings with the refractive index structure given in Eq. (31). The core-tocore transmission intensity spectrum was calculated by the IFOgratings software. The figure shows couplings to four different cladding modes, $\mathrm{LP}_{02-05}$.

In the last example, we demonstrate the reconstruction method described in Subsection 3.C. We did not observe a dependence of the reconstruction quality on the specific grating profiles that were chosen as long as the auxiliary grating was significantly shorter than the interrogated grating and the coupling efficiency of both gratings was lower than about $20 \%$. In our example we have reconstructed a cascaded structure of weak Gaussian and uniform gratings from only the amplitude of the core-to-core transmission spectrum of the structure. Since the coupling between the core and the cladding modes of the two gratings is weak, only the core-to-core transmission amplitude of the total structure is needed, as explained in Subsections 3.C and Section 4. The length of the uniform grating was 15 times longer than the length of the Gaussian grating. The intensity of the core-to-core transmission spectrum was calculated by using the IFO-gratings software written by Optiwave Corporation. The IFO-gratings software simulates the coupling to all the different cladding modes of the grating. The refractive indices of the core and cladding were 1.458 and 1.45 , respectively. The radii of the core and cladding were 2.625 and $62.5 \mu \mathrm{m}$. The grating period was $\Lambda=500 \mu \mathrm{m}$, and the refractive index amplitude was equal to

$$
n_{1}(z)= \begin{cases}1.2 \times 10^{-5}, & 0<z<0.15 \mathrm{~m} \\ 2.25 \times 10^{-4} \exp \left[-(z-0.325) / 1.6 \times 10^{-5}\right], & 0.32 \mathrm{~m}<z<0.33 \mathrm{~m}\end{cases}
$$

The intensity of the core-to-core transmission function of the structure was calculated with a resolution of $0.3 \mathrm{~nm}$ and a bandwidth of $300 \mathrm{~nm}$. Figure 7 shows the calculated intensity of the core-to-core transmission function. The figure shows a coupling to four different cladding modes, $\mathrm{LP}_{02-05}$. We used only the spectrum formed by coupling to the $\mathrm{LP}_{05}$ cladding mode to reconstruct the grating. Therefore, we used only the transmission function at the wavelength region $1520-1620 \mathrm{~nm}$, as marked in Fig. 7. We added to each calculated point in the intensity function $\left|a_{\text {tot }}(k)\right|^{2}$ a random variable, which represented the noise added in an experiment. The random variables were independent and had a Gaussian distribution with a zero mean and a standard deviation of $10^{-3}$. The core-to-core transmission function was represented as a function of the wavenumber detuning $k$ by using the following relation: $k=\pi\left[\Delta n_{\text {eff }}(\lambda) / \lambda-\Delta n_{\text {eff }}\left(\lambda_{c}\right) / \lambda_{c}\right]$, where $\Delta n_{\text {eff }}$ is the difference in the effective indices of the core mode and cladding mode, $\lambda$ is the wavelength, and $\lambda_{c}$ $=n_{\text {eff }}\left(\lambda_{c}\right) \Lambda$ is the central wavelength of the grating. We used the SHIO algorithm to reconstruct the phase of the function $b_{\text {tot }}(k)$ from its amplitude. The grating profile was reconstructed from the core-to-cladding transmission function $b_{\text {tot }}(k)$ by using Eqs. (10). The spatial resolution of the reconstructed profile was about $4 \mathrm{~mm}$. Figure 8 shows the reconstruction of the grating from the noisy transmission intensity (solid curve). The result is compared with a reconstruction from the accurate transmission intensity, which was not contaminated by noise (dashed curve). The figure shows that a very good reconstruction of the grating was obtained. The main source for the small errors in the reconstruction is the approxima- tions made in expressions (10) and (15).

The spatial resolution of the reconstruction is given by $\Delta z=\left|\Delta n_{\text {eff }}\left(\lambda_{\min }\right) / \lambda_{\min }-\Delta n_{\text {eff }}\left(\lambda_{\max }\right) / \lambda_{\max }\right|^{-1}$, where $\lambda_{\max }$ and $\lambda_{\text {min }}$ are the maximum and minimum wavelengths of the spectral measurement, respectively. In our example, the coupling to more than a single cladding mode limits the spectral bandwidth used to reconstruct the grating and, thus, also limits the spatial resolution of the grating reconstruction. In our last example, we obtained a recon-

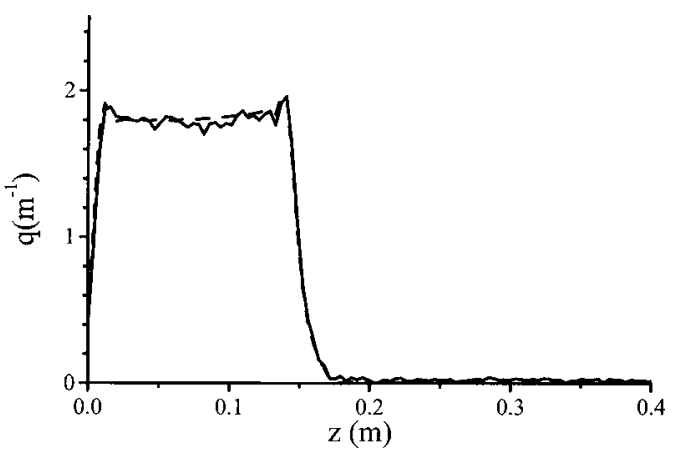

Fig. 8. Reconstruction of the long (interrogated) uniform grating from the core-to-core transmission intensity shown in Fig. 7 at the frequency region of $1520-1620 \mathrm{~nm}$. The reconstruction was obtained from the noisy (solid curve) and accurate (dashed curve) transmission intensities by using the phase-retrieval method. The noisy transmission spectrum was obtained by adding to each calculated point in the transmission intensity an independent random variable that had a Gaussian distribution with a zero mean and a standard deviation of $1 \times 10^{-3}$. The reconstruction of the gratings from the core-to-core transmission intensity spectrum was possible, since the two reconstructed gratings were weak and therefore the Born approximation could be used. 
struction resolution of $4 \mathrm{~mm}$ for a grating with a period of $0.5 \mathrm{~mm}$. We note that the reconstruction resolution is only eight times larger than the grating periodicity. In comparison, the FBG that was interrogated in Ref. 17 was measured with a spatial resolution that was equal to about 140 times the grating period. When the LPG is used as a distributed sensor, the resolution may be improved by choosing a fiber with a high $\Delta n_{\text {eff }}$ whose cladding modes have large effective-refractive-index differences between them. The resolution can also be improved by optimizing the choice of the grating periodicity.

\section{SUMMARY}

In this paper we have shown theoretically that the structure of LPGs can be reconstructed from their complex core-to-core transmission spectra. To obtain a unique reconstruction, an additional auxiliary grating should be written in cascade to the interrogated grating such that the spatial gap between the gratings is sufficiently large. We consider the reconstruction in three cases, in which different input data are used. For each case, a different minimum gap is required to ensure a unique reconstruction. We found that in the second and the third cases the reconstruction is not sensitive to noise when the length of the auxiliary grating is significantly shorter than the length of the interrogated grating. Two methods for extracting the core-to-cladding transmission spectra from the measured core-to-core transmission spectra are demonstrated. The first is based on a phase-retrieval algorithm, and the second is based on a deconvolution algorithm. Our results are summarized in Table 1.

Our work enables the reconstruction of LPGs using only the core-to-core transmission spectrum. Such a method can enable reconstructing, for the first time, the structure of LPGs from their measured spectra, as performed for FBGs. The reconstruction technique for LPGs may be useful for improving the writing process of LPGs and for developing novel distributed sensors. The resolution of the reconstruction is determined by the maximum bandwidth where there is coupling to only a single cladding mode. In our example we obtained a spatial reconstruction resolution of $4 \mathrm{~mm}$ for a grating with a period of $0.5 \mathrm{~mm}$. The resolution may be enhanced by using fibers with a high effective-refractive-index difference between the core and the cladding modes and with a high spectral spacing between the cladding modes. However, a resolution better than the grating periodicity cannot be obtained owing to the use of coupled-mode theory.

\section{APPENDIX A}

In this appendix we briefly describe the SHIO algorithm. The SHIO algorithm is composed of two parts: The first part converges to a local minimum solution, and the second part enables emerging from the local minimum solution. In Ref. 29 the algorithm was developed for the phase-retrieval problem of two-dimensional real signals. Although the signal in our case is a complex onedimensional signal, it can be easily shown that the derivation of the algorithm in Ref. 29 remains valid.

We define the functions $\beta_{n}^{\text {in, }, D}(\tau)$ and $\beta_{n}^{\text {out }, D}(\tau)$ as

$$
\beta_{n}^{\{\text {in }, \text { out }\}, D}(\tau)= \begin{cases}\beta_{n}^{\{\text {in }, \text { out }\}}(\tau), & \tau \in D \\ 0, & \tau \notin D\end{cases}
$$

and the functions $\beta_{n}^{\text {in, } N D}(\tau)$ and $\beta_{n}^{\text {out }, N D}(\tau)$ as

$$
\beta_{n}^{\{\text {in }, \text { out }\}, N D}(\tau)=\beta_{n}^{\{\text {in }, \text { out }\}}(\tau)-\beta_{n}^{\{\text {in,out }\}, D}(\tau),
$$

where the functions $\beta_{n}^{\{\text {in,out }\}}$ are the input and output functions of the $n$th iteration of the algorithm, as defined in Subsection 4.A. The inverse Fourier transforms of the functions $\beta_{n}^{\{i n, o u t\}, D}(\tau)$ and $\beta_{n}^{\{i n, o u t\}, D}(\tau)$ are given by $b_{n}^{\{\text {in,out }\}, D}(k)$ and $b_{n}^{\{\text {in out }\}, N D}(k)$, respectively. The converging part of the SHIO algorithm is described by the following relations:

$$
\begin{gathered}
b_{n}^{\text {out }}(k)=\frac{b_{n}^{\text {in }}(k)}{\left|b_{n}^{\text {in }}(k)\right|}\left|b_{\mathrm{tot}}(k)\right|, \\
b_{n+1}^{\text {in }}(k)=b_{n}^{\text {in, }, N D}(k)+b_{n}^{\text {out }, D}(k)+i \gamma \frac{1}{\left|b_{\mathrm{tot}}(k)\right|^{2}} \\
\times \operatorname{Im}\left\{b_{n}^{\text {out }}(k)\left[b_{n}^{\text {out }, N D}(k)\right]^{*}\right\} b_{n}^{\text {out }}(k) .
\end{gathered}
$$

The convergence parameter is defined by

$$
C_{n}=\left[\frac{\sum_{m}\left(\operatorname{Im}\left\{b_{n}^{\text {out }}\left(k_{m}\right)\left[b_{n}^{\text {out }, N D}\left(k_{m}\right)\right]^{*}\right\}\right)^{2}}{\sum_{m}\left|b_{n}^{\text {out }}\left(k_{m}\right)\right|^{2} \sum_{m}\left|b_{n}^{\text {out }, N D}\left(k_{m}\right)\right|^{2}}\right] / 2 .
$$

It is shown in Ref. 29 that, when the algorithm converges, $\left|b_{n+1}^{i n}(k)-b_{n}^{i n}(k)\right|$ and $C_{n}$ approach zero, and, therefore, at the convergence of Eq. (A3), we obtain

$$
\operatorname{Im}\left\{b_{n}^{\text {out }}(k)\left[b_{n}^{\text {out }, N D}(k)\right]^{*}\right\}=0 .
$$

Practically, the iterations are stopped when the parameter $C_{n}$ is less than a predetermined level $\delta$, where $\delta \ll 1$. We used in our simulations $\delta=10^{-4}$, as in Ref. 29 .

According to Eq. (A5), the difference between the phases of the functions $b_{n}^{\text {out }}(k)$ and $b_{n}^{\text {out }, N D}(k)$ is either zero or $\pi$ for each value of $k$ in which the functions $b_{n}^{\text {out }}(k)$ and $b_{n}^{\text {out }, N D}(k)$ are not equal to zero. We note that when $b_{n}^{\text {out }, N D}(k)$ is identically equal to zero, the function $b_{n}^{\text {out }}(k)$ is the solution of the phase-retrieval problem, defined in Eqs. (18) and (19). In addition, it is shown in Ref. 30 that when $b_{n}^{\text {out }, N D}(k)$ is not identically equal to zero there exists at least one value of $k$, where the phases of the functions $b_{n}^{\text {out }}(k)$ and $b_{n}^{\text {out }, N D}(k)$ are equal. We denote by $\left\{k_{n}\right\}$ the group of all the wavenumbers for which the function $b_{n}^{\text {out }}\left(k_{n}\right)$ has the same phase as the function $b_{n}^{\text {out }, N D}\left(k_{n}\right)$ and define a function $r\left(k_{n}\right)$ as

$$
r\left(k_{n}\right)=\frac{\left|b_{n}^{\text {in }}\left(k_{n}\right)\right|}{\left|b_{n}^{\text {out }, N D}\left(k_{n}\right)\right|} .
$$

The smallest and second-smallest values of $r\left(k_{n}\right)$ are denoted by $r_{1}$ and $r_{2}$. Using the above definitions, we can describe the emerging part of the SHIO algorithm by the following equation:

$$
b_{n+1}^{\text {in }}(k)=b_{n}^{\text {in }}(k)-\left(\frac{r_{1}+r_{2}}{2}\right) b_{n}^{\text {out }, N D}(k) .
$$


The SHIO algorithm is then performed using the following steps:

1. Apply Eqs. (A3) until $C_{m}<\delta$.

2. Apply Eq. (A7) once.

3. Return to step 1 .

\section{ACKNOWLEDGMENTS}

The help of Michael Rabinovich and Amit Rappel with the numerical simulations of the inverse-scattering algorithm is acknowledged. The authors may be reached by e-mail as follows: Amir Rosenthal, eeamir@tx.technion.ac.il, and Moshe Horowitz, horowitz@ee.technion.ac.il.

\section{REFERENCES}

1. K. O. Hill and G. Meltz, "Fiber Bragg grating technology fundamentals and overview," J. Lightwave Technol. 15, 1263-1276 (1997)

2. D. B. Stegall and T. Erdogan, "Dispersion control with use of long-period fiber gratings," J. Opt. Soc. Am. A 17, 304-312 (2000).

3. B. H. Lee and J. Nishii, "Dependence of fringe spacing on the grating separation in a long-period fiber grating pair," Appl. Opt. 38, 3450-3459 (1999).

4. T. Allsop, T. Earthrowl, R. Reeves, D. J. Webb, and I. Bennion, "The interrogation and multiplexing of long period grating curvature sensors using a Bragg grating based, derivative spectroscopy technique," Meas. Sci. Technol. 15, 44-48 (2004).

5. E. Peral, J. Capmany, and J. Marti, "Iterative solution to the Gel'fand-Levitan-Marchenko coupled equations and application to synthesis of fiber gratings," IEEE J. Quantum Electron. 32, 2078-2084 (1996).

6. R. Feced, M. N. Zervas, and M. A. Muriel, "An efficient inverse scattering algorithm for the design of nonuniform fiber Bragg gratings," IEEE J. Quantum Electron. 35, 1105-1115 (1999).

7. J. Skaar, L. Wang, and T. Erdogan, "On the synthesis of fiber Bragg gratings by layer peeling," J. Lightwave Technol. 37, 165-173 (2001).

8. A. Rosenthal and M. Horowitz, "Inverse scattering algorithm for reconstructing strongly reflecting fiber Bragg gratings," IEEE J. Quantum Electron. 39, 1018-1026 (2003).

9. M. Sumetsky, P. I. Reyes, P. S. Westbrook, N. M. Litchinitser, B. J. Eggleton, Y. Li, R. Deshmukh, and C. Soccolich, "Group-delay ripple correction in chirped fiber Bragg gratings," Opt. Lett. 28, 777-779 (2003).

10. S. Keren and M. Horowitz, "Distributed three-dimensional fiber Bragg grating refractometer for biochemical sensing," Opt. Lett. 28, 2037-2039 (2003).

11. V. Goloborodko, S. Keren, A. Rosenthal, B. Levit, and M. Horowitz, "Measuring temperature profiles in high-power optical fiber components," Appl. Opt. 42, 2284-2288 (2003).

12. R. Feced and M. N. Zervas, "Efficient inverse scattering algorithm for the design of grating-assisted codirectional mode couplers," J. Opt. Soc. Am. A 17, 1573-1582 (2000).

13. L. Wang and T. Erdogan, "Layer peeling algorithm for reconstruction of long-period fiber gratings," Electron. Lett. 37, 154-156 (2001).

14. J. K. Brenne and J. Skaar, "Design of grating-assisted co-directional couplers with discrete inverse-scattering algorithms," J. Lightwave Technol. 21, 254-263 (2003).

15. I. Petermann, J. Skaar, B. Sahlgren, R. Stubbe, and A. Friberg, "Characterization of fiber Bragg gratings by use of optical coherence-domain reflectometry," J. Lightwave Technol. 17, 2371-2378 (1999).

16. S. Keren and M. Horowitz, "Interrogation of fiber gratings by use of low-coherence spectral interferometry of noiselike pulses," Opt. Lett. 26, 328-330 (2001).

17. S. Keren, A. Rosenthal, and M. Horowitz, "Measuring the structure of highly reflecting fiber Bragg gratings," IEEE Photon. Technol. Lett. 15, 575-577 (2003).

18. K. Thyagarajan, M. Das, and M. N. Satyanarayan, "A simple and direct method to estimate long period grating parameters," Opt. Commun. 218, 67-72 (2003).

19. T. Erdogan, "Fiber grating spectra," J. Lightwave Technol. 15, 1277-1294 (1997)

20. B. H. Lee and U. C. Paek, "Multislit interpretation of cascaded fiber gratings," J. Lightwave Technol. 20, 1750-1761 (2002).

21. A. Rosenthal and M. Horowitz, "Reconstruction of a fiber Bragg grating from noisy reflection data," J. Opt. Soc. Am. A 22, 84-92 (2004).

22. M. J. Ablowitz and H. Segur, Solitons and the Inverse Scattering Transform (Society for Applied Mathematics, 1981).

23. T. R. Crimmins and J. R. Fienup, "Uniqueness of phase retrieval for functions with sufficiently disconnected support,” J. Opt. Soc. Am. 73, 218-221 (1983).

24. G. H. Song, "Theory of symmetry in optical filter responses,” J. Opt. Soc. Am. A 11, 2027-2037 (1994).

25. T. Isernia, G. Leone, R. Pierri, and F. Soldovieri, "Role of support information and zero locations in phase retrieval by a quadratic approach,” J. Opt. Soc. Am. A 16, 1845-1856 (1999).

26. M. Carmody, E. Landree, L. D. Marks, and K. L. Merkle, "Determination of the current density distribution in Josephson junctions," Physica C 315, 145-153 (1999).

27. M. Nieto-Vesperinas, R. Navarro, and F. J. Fuentes, "Performance of a simulated-anneling algorithm for phase retrieval," J. Opt. Soc. Am. A 5, 30-38 (1988).

28. J. R. Fienup, "Phase retrieval algorithms: a comparison," Appl. Opt. 21, 2758-2769 (1982).

29. H. Takajo, T. Shizuma, T. Takahashi, and S. Takahata, "Reconstruction of an object from its noisy Fourier modulus: ideal estimate of the object to be reconstructed and a method that attempts to find that estimate," Appl. Opt. 38, 5568-5576 (1999).

30. H. Takajo, T. Takahashi, R. Ueda, and M. Taninaka, "Study on the convergence property of the hybrid input-output algorithm used for phase retrieval," J. Opt. Soc. Am. A $\mathbf{1 5}$, 2849-2861 (1998).

31. A. L. Oppenheim and R. W. Schafer, Discrete-Time Signal Processing (Prentice Hall, 1999). 